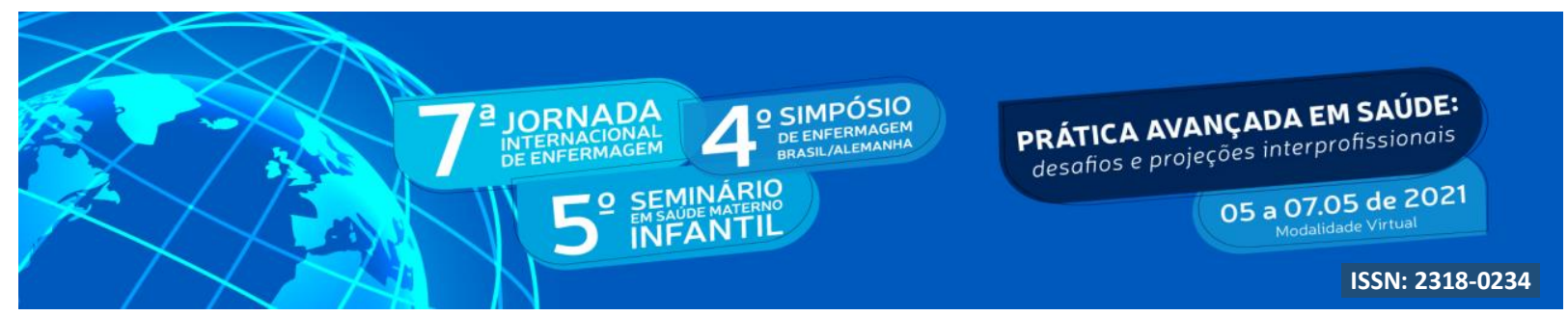

DOI: http://doi.org/10.48195/jie2021-179

\title{
FLUXOGRAMA PARA GESTANTES NA REDE DE CUIDADOS EM SAÚDE
}

\section{Jessica Morais da Silva Paim ${ }^{1}$; Amanda Ruiz Forgiarini² ${ }^{2}$ Francine Casarin ${ }^{4}$; Francine Santellano Susin ${ }^{5}$; Patricia Cristiane da Costa Dutra ${ }^{6}$; Mara Regina Caino Teixeira Marchiori ${ }^{7 .}$}

\begin{abstract}
RESUMO
O Sistema Único de Saúde, tem por base conceitos como democracia, emancipação e igualdade, teve como objetivo ser um sistema que promovesse acesso ao cuidado em saúde por qualquer indivíduo quando esse precise, sem importar a categoria do cuidado. Objetiva-se relatar a experiência à respeito da criação de um fluxograma sobre o pré-natal. Trata-se de um relato de experiência vivenciada no nono semestre do curso de enfermagem. Conclui-se que aliar a atividade teórica da graduação com a inserção do acadêmico ainda na formação assim de enriquecer as vivencias do mesmo, fazendo com que pense e haja criticamente de acordo com as necessidades da população.
\end{abstract}

Palavras-chave: Enfermagem; Gravidez; SUS; Trabalho.

\begin{abstract}
The Unified Health System, based on concepts such as democracy, emancipation and equality, aimed to be a system that promotes access to health care by any individual when he needs it, regardless of the category of care. The objective is to report the experience of creating a flowchart about prenatal care. This is an experience report lived in the ninth semester of the nursing course. It is concluded that combining the theoretical activity of graduation with the insertion of the student still in training enriches their experiences, making them think and act critically according to the needs of the population.
\end{abstract}

Key Words: Nursing; Pregnancy; SUS; Work.

\section{INTRODUÇÃO}

O Sistema Único de Saúde (SUS), baseado em conceitos como democracia, emancipação e igualdade, foi criado afim de promover acesso a saúde a individuo que

\footnotetext{
${ }^{1}$ Estudante do Curso de Enfermagem. UFN. E-mail: jessica.paim@ufn.edu.br

${ }^{2}$ Estudante do Curso de Enfermagem. UFN. E-mail: mandiruiz@ hotmail.com.br

${ }^{4}$ Enfermeira: fracasarin@hotmail.com

${ }^{5}$ Estudante do Curso de Enfermagem. UFN. E-mail: franssusin@gmail.com

${ }^{6}$ Enfermeira: esf.abv@gmail.com

${ }^{7}$ Orientador. Professora Curso Enfermagem. UFN. E-mail: mara.marc@ hotmail.com
} 


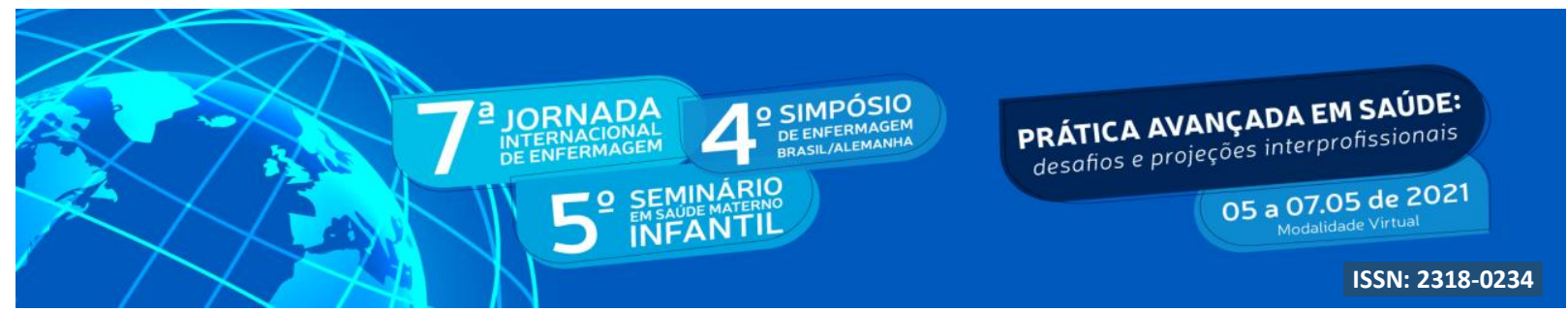

necessitem de cuidados, não importando a categoria, básico, intermediário ou grave. O Brasil com a descentralização de poderes e recursos, ampliou as oportunidades de prestação e obtenção de serviços e ações, e impactou no nível da saúde de maneira uniforme em todas as esferas do governo (PAIM, 2018).

Embora a gestão governamental do trabalho nas três áreas imponha limitações e cause insatisfação com os serviços públicos, o processo de implantação do SUS tem despertado o entusiasmo e o comprometimento dos trabalhadores da saúde em contato com a Secretaria e o Ministério da Saúde (PAIM, 2018).

Para que se tenha um bom andamento dos serviços de saúde, entra o processo de trabalho em saúde, que é compreendido como uma soma de intervenções realizadas por trabalhadores, tendo as pessoas, famílias e comunidades como objeto de trabalho e suas competências e técnicas são seus instrumentos e destes resultam a atenção à saúde de maneira integral e de maior qualidade. Neste contexto, os profissionais de saúde possuem um papel fundamental, sendo o elo entre os objetos de trabalho e os métodos (FONTANA et al., 2016; CARRAPATO et al., 2018).

Além disso, o processo de trabalho em saúde tem como objetivo conseguir cumprir os princípios e diretrizes do Sistema Único de Saúde (SUS) a fim de auxiliar os profissionais de saúde a atender as necessidades das pessoas e comunidades, garantir acesso ao cuidado humanizado, integral e de qualidade. Percebe-se também que além do que já foi citado acima, os processos de trabalho são essenciais para a organização dos serviços (TABILE et al., 2015).

Dentre os métodos utilizados no processo de trabalho temos o fluxograma, que é método de interpretação gráfica que utiliza símbolos estabelecidos, possibilitando a compreensão e análise nítida e detalhada de algum processo. Sendo assim, entende-se que o fluxograma permite que o profissional preste a assistência de maneira mais clara e efetiva (TABILE et al., 2015).

\section{OBJETIVO}

Objetiva-se relatar a experiência dos discentes do nono semestre do curso de enfermagem, referente a teorização e criação de um fluxograma para atender as necessidades levantadas pelos acadêmicos em relação no Sistema Único de Saúde ao relatar os 


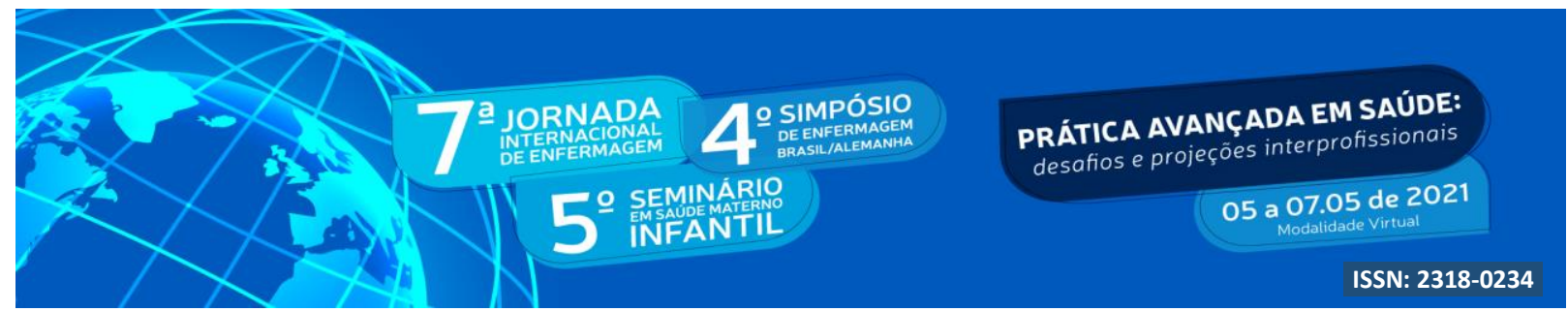

encaminhamentos de gestantes em uma unidade de saúde de uma cidade da região central do Rio Grande do Sul.

\section{METODOLOGIA}

O presente trabalho é de caráter descritivo, do tipo relato de experiência, realizado a partir da vivência discente durante o estágio curricular supervisionado, em conjunto com atividade avaliativa desenvolvida a partir da disciplina de Processo de Trabalho em Saúde, do Curso de Enfermagem da Universidade Franciscana. Realizado no período de julho a dezembro de 2021. De acordo com Gil (2008), o relato de experiência proporciona ao pesquisador relatar suas experiências e vivências e contribui de forma relevante para sua área de atuação.

\section{RESULTADOS E DISCUSSÃO}

O SUS conta com uma rede de instituições de ensino e pesquisa, como universidades, escolas de saúde pública e escolas que interagem com secretarias estaduais e municipais, Ministério da Saúde, instituições e fundações. Essa rede contribui para o desenvolvimento sustentável da organização, possibilita que um grupo de pessoas desenvolvam conhecimentos, habilidades e valores relacionados aos princípios e diretrizes do SUS. Muitos deles apoiam o SUS mesmo em circunstâncias difíceis, se tornando essenciais para a efetiva funcionalidade das unidades (PAIM, 2018).

Vale ressaltar que a atenção primária à saúde conecta cerca de $60 \%$ da população brasileira com grupos de saúde da família. O país avançou no desenvolvimento de sistemas de informação em saúde, como sistemas de informação relacionados a questões de mortalidade, hospitalização e notificação, importantes para o monitoramento e avaliação de políticas, planos e programas. Vale ressaltar, ainda, que a falta de compreensão do direito à saúde possibilita a difusão dessa conquista na sociedade, seja na concretização da cidadania, na mídia, ou no processo de judicialização do ponto de vista cultural (PAIM, 2018).

Quando utilizamos o fluxograma no processo de trabalho na saúde, podemos observar como os objetos se conectam e se relacionam, podendo identificar com maior facilidade as suas fragilidades. Outro ponto positivo da utilização do fluxograma é a fácil interpretação por todas 


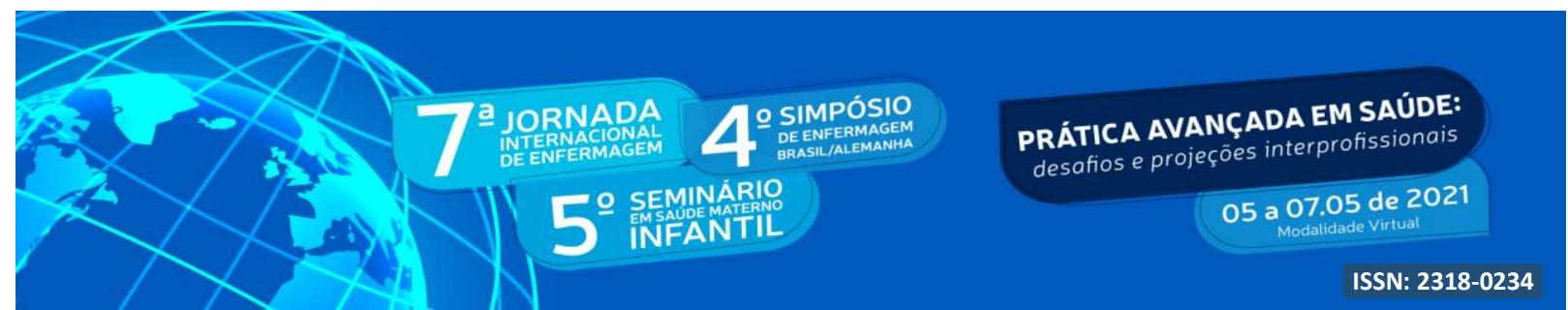

as partes envolvidas no processo de trabalho. Por este motivo, este método é muito utilizado na atenção primária em saúde (AZEVEDO, 2016).

Sendo assim, durante as aulas práticas e os estágios na atenção primária as acadêmicas de enfermagem levantaram a necessidade e a dificuldade que percebiam no atendimento as gestantes de uma Estratégia Saúda Família (ESF). Então após discussões, criou-se o fluxograma que permeia todo o atendimento, desde a descoberta da gestação, até o momento do parto.

Este fluxograma foi pensado em conjunto com a equipe da ESF, sendo a líder, a enfermeira, visando facilitar o cotidiano dos acadêmicos, dos agentes comunitários de saúde, profissionais da unidade e principalmente a gestante usuária do serviço. Sendo um instrumento utilizado durante o pré-natal, permitindo a visualização do seu percurso pela rede de atenção e como se dará o acompanhamento da mesma. Sabemos que esta ainda é uma dificuldade nos serviços pois, geralmente o usuário não conhece os caminhos aos quais vai percorrer. Então, percebemos o fluxograma como um método simples e eficaz de organização e orientação de um processo em saúde.

Abaixo, na figura 1, segue o fluxograma desenvolvido pelas autoras afim de exemplicar os encaminhamentos que as gestantes devem receber de acordo com o transcorrer do período gravídico. 


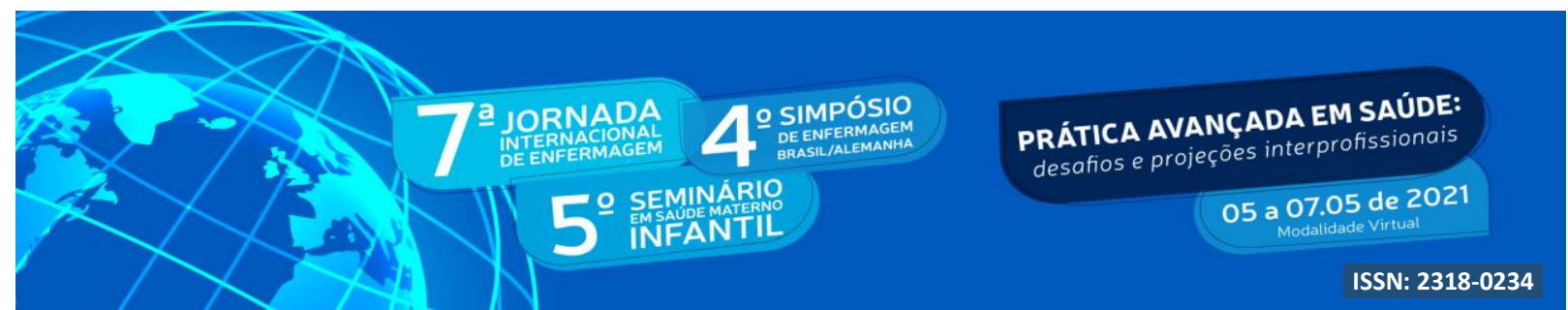

Figura 1: Fluxograma.

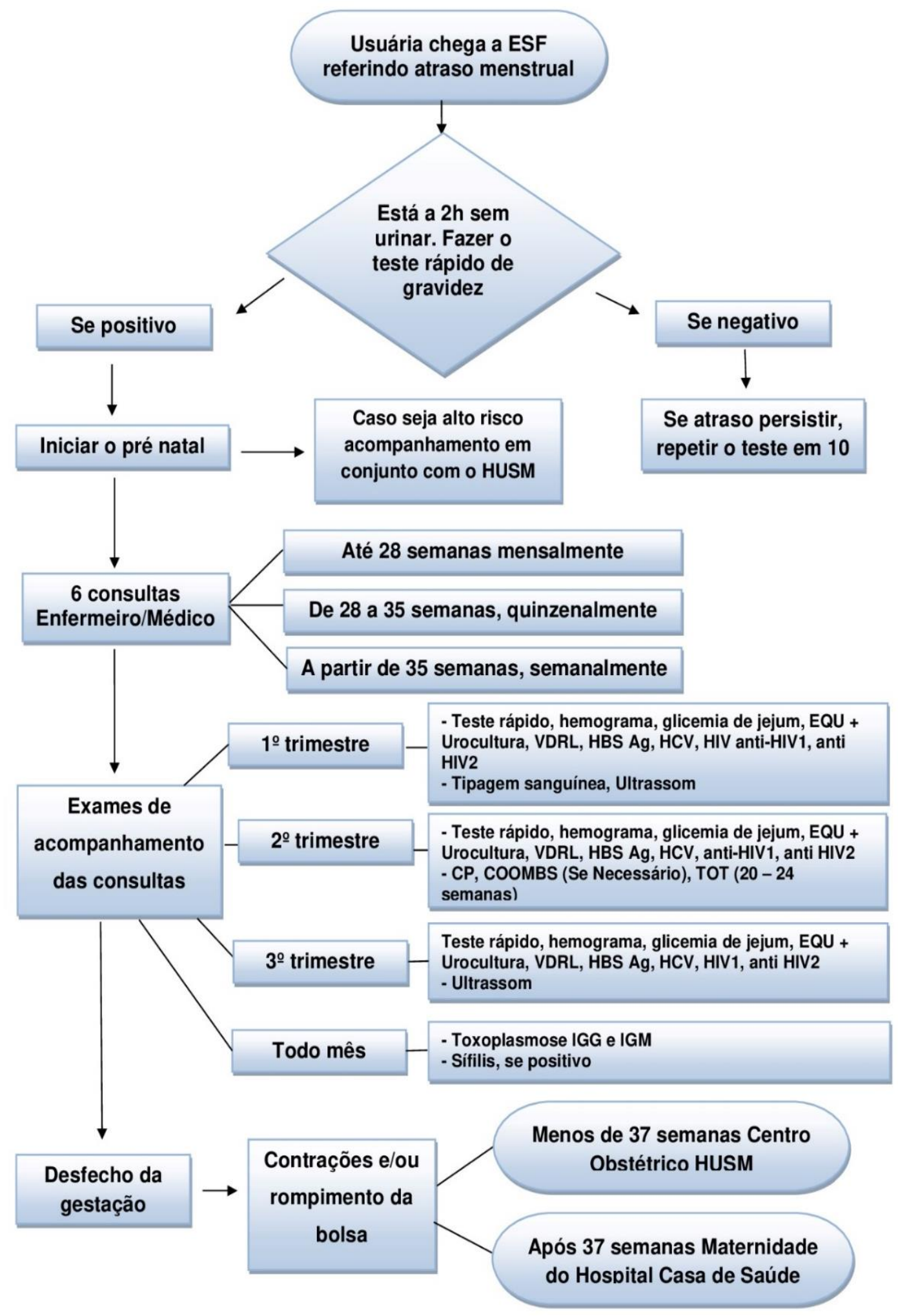

Fonte: Construção dos autores 


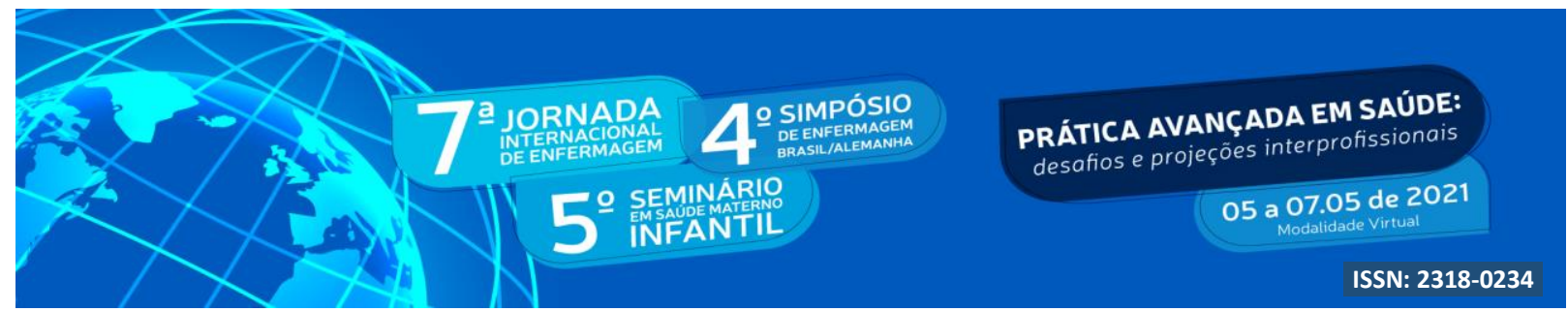

Destaca-se que no fluxograma, de acordo com o desfecho da gestação, há exames para realizar de acordo com o trimestre e semanas de gestação, além é claro, de para quais locais a gestante se direcionará caso entre em trabalho de parto, havendo contração ou rompimento da bolsa. Importante salientar que, esse fluxograma exemplifica o trajeto de mulheres com gestação de risco habitual e alto risco. Percebe-se também que pelo fluxograma acompanhamos desde a confirmação da gravidez até o nascimento da criança.

Ao olhar mais atentamente para o fluxograma criado, percebe-se também que é utilizada uma linguagem acessível a população no que tange as informações necessárias ao tema exposto, além de ser um método para que tantos profissionais como acadêmicos possam consultar caso haja alguma dúvida sobre exames ou para onde encaminhar as gestantes. Ao se valer do fluxograma como ferramenta de informação e consulta, o importante é que os indivíduos de saúde que fazem uso, procurem mantê-lo atualizado para que assim possa ser utilizado de maneira correta.

\section{CONCLUSÃO}

Após expor o fluxograma e ao tema que ele foi utilizado, percebe-se a importância da equipe de saúde estar atenta aos problemas e dificuldades que a população que frequenta a unidade de saúde enfrenta, e, após esse diagnóstico, a maneira como conduzir o planejamento para a resolução da dificuldade encontrada também deve ser feito afim de abranger a maior parte da população possível alem de, se for para explicação, de fácil entendimento para a população em geral.

Importante ressaltar a inserção dos acadêmicos em processo formativo, pois assim, há um impacto positivo visto que irá ser aliado a teoria que se estuda durante a formação bem como, com a prática profissional diretamente relacionada ao tema, culminando assim em uma formação de um profissional crítico e que desde sua construção percebe a importância do processo de trabalho na saúde afim de desenvolver um trabalho de acordo com as demandas da população. 


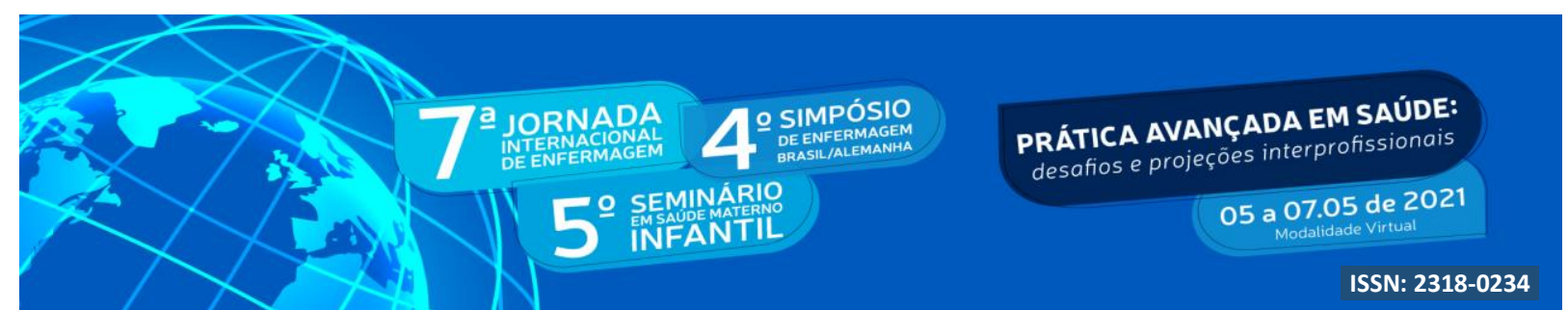

\section{REFERÊNCIAS}

CARRAPATO, J. F. L; CASTANHEIRA, E. R. L; PLACIDELI, N. Percepções dos profissionais de saúde da atenção primária sobre qualidade no processo de trabalho. Saúde e Sociedade [online]. 2018, v. 27, n. 2

FONTANA, K. C; LACERDA, J. T. MACHADO, P. M. de O. O processo de trabalho na Atenção Básica à saúde: avaliação da gestão. Saúde em Debate [online]. 2016, v. 40, n. 110, pp. 64-80. Disponível em: <https://doi.org/10.1590/0103-1104201611005>. ISSN 2358-2898.

GIL, A. C. Como elaborar projetos de pesquisa. 5. ed. São Paulo: Atlas, 2008.

PAIM, J. S. Sitema único de saúde (SUS) aos 30 anos. Ciência \& Saúde Coletiva, 23(6):17231728,2018

TABILE, P. M, et.al. A importância do fluxograma para o trabalho da saúde da família na visão do projeto pet-saúde. Revista Eletrônica Gestão \& Saúde ISSN: 1982-4785.

AZEVEDO, I. C. G. Fluxograma como ferramenta de mapeamento de processo no controle de qualidade de uma indústria de confecção. XII Congresso Nacional de Excelência em Gestão. 2016.. 